\title{
INDEX IMAGINVM
}

In fronte libri CAPVT MARMOREVM, paene intactum Ostiae ut dicunt repertum, nunc Londinii in museo kensingtoniano a v. cl. Ashley Ponsonby publice propositum, tragicam spirans gravitatem :

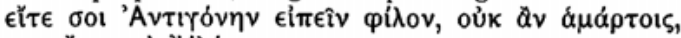

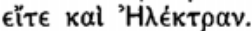

plura de hoc capite exposita invenies ab Adolfo Michaelis archaeol. zeitung 1880 p. $75-83$ et anc. marbles in Great Britain p. 484.

In fronte praefationis Orestes Pylade comitante Electrae velatae urnam offerens, PICTVRA VRNAE APVLAE, servatae in museo vindobonensi $(\mathrm{IV}, 69)$. ediderunt et interpretati sunt Labordius vases Lamberg I tab. 8, I. de Witte et C. Lenormantius élite céramogr. II tab. 79, Overbeckius bildwerke tab. 29, 6. novam delineationem suppeditavit O. Benndorfius.

In fronte vita e CAPVT SOPHOCLIS, pars statuae marmoreae musei lateranensis (n. 237 ), prope Tarracinam inventae, de qua legere iuvabit quae disputaverunt Welckerus alte denkmäler I p. 455-484, Wieselerus götting. gel. anz. 1848 p. $1220-12 j 1$, Benndorfius et Schoenius ant. bildw. des lateran. mus. p. $153-159$.

Sub inscriptione fabulae ORESTES Er ELECTRA E MARMORE, egregium artis antiquae pulchritudinem gravitate temperantis opus, e schola pasitelea profectum, quod Neapoli in museo publico conspicitur. ediderunt et cxplicuerunt Radulphus Rochettius mon. inéd. tab. 33, 1. p. 166, Overbeckius bildw. tab. 28, 6. p. (992, Iahnius ber. d. sächs. ges. 1861 tab. 4, 1. p. 103-109, Kekuléus gruppe des Menelaos tab. 2, 1. p. 25. 32.

In fronte fabulae PICTVRA AMPHORAE LVCANAE Neapoli in museo publico servatae (n. 1984 Heyd.). Apollo in omphalo sedens Oresten, qui cum Pylade oraculum consultum accessit, patrem ulcisci iubet, praesentibus et Pythia in tripode seclente et famula. quam Electram quidam perhibent. picturae plerumque ad Oresten a deo expiatum relatae (cf. Rad. Rochettius mon. inéd. tab. 37. p. 187, Overbeckius bildw. tab. 29, 11. p. 715) rectam interpretationem primus proposuit Boetticherus archaeol. zeit. 1860 tab. 138,1 . p. $49-61$.

In fronte supplementi caedes Aegisthi depicta in OLLA VOL CENTANA musei berolinensis (n. 1007), quam ediderunt Gerhardus etrusk. und kampan. vasenb. tab. 24. p. 35 et Overbeckius bildw. tab. 28, 10. p. 695. de explicando Electrae pone Aegisthum adstantis gestu in varias partes discesserunt praeter Gerhardum Welckerus alte denkm. V p. $292-297$, Iahnius archacolog. zeit. 1860 p. 43 , Benndorfius ann. dell' inst. 1865 p. $217-219$.

In fine libelli PICTVRaE ollae caeretanaE Vindobonae in museo austriaco servatae, quam cditam mon. dell' inst. VIII pl. $1 \bar{j}$ explicavit Benndorfius ann. 1865 p. $212-216$. in parte antica Aegisthum e solio Atridarum labentem interficit Orestes, effugiente Chrysothemide; ab altera parte Clytaemnestra accurrit bipenni armata, quam arcet Talthybius

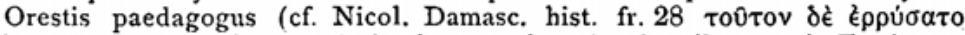

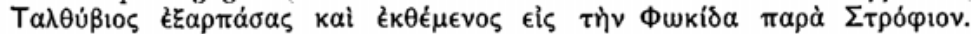
Dict. 6, 2 Talthybius Oresten Agamemnonis filium manibus Aegisthi ereptum Idomeneo, qui apud Corinthum agebat, tradit.). 